Cahiers de philosophie de l'université de

\title{
Cosmologie et psychologie des crises économiques chez Gabriel Tarde
}

Pierre Montebello

\section{OpenEdition}

1 Journals

Édition électronique

URL : https://journals.openedition.org/cpuc/323

DOI : $10.4000 /$ cpuc.323

ISSN : 2677-6529

Éditeur

Presses universitaires de Caen

\section{Édition imprimée}

Date de publication : 31 décembre 2017

Pagination : 123-134

ISBN : 978-2-84133-859-7

ISSN : $1282-6545$

\section{Référence électronique}

Pierre Montebello, "Cosmologie et psychologie des crises économiques chez Gabriel Tarde », Cahiers de philosophie de l'université de Caen [En ligne], 54 | 2017, mis en ligne le 01 février 2019, consulté le 02 février 2023. URL : http://journals.openedition.org/cpuc/323 ; DOl : https://doi.org/10.4000/cpuc.323

\section{(c) $(1) \otimes$}

Creative Commons - Attribution - Pas d'Utilisation Commerciale 4.0 International - CC BY-NC 4.0 https://creativecommons.org/licenses/by-nc/4.0/ 


\section{Cosmologie et psychologie des crises économiques chez Gabriel Tarde}

\footnotetext{
$\mathrm{O}$ N CONNAÎT LA CÉLÈbRE AFFIRMATION de Bruno Latour:

Quel soulagement, nous ne sommes pas «en» société, pas plus que nous sommes «dans » la nature. Le social n'est pas un horizon implacable à l'intérieur duquel viendrait s'inscrire chacun de nos gestes; la société n'a pas cette présence omniprésente, omnisciente qui surveille chacun de nos mouvements et sonde chacune de nos pensées les plus secrètes comme le Dieu omnipotent de nos anciens catéchismes ${ }^{1}$.
}

Et de se référer à Tarde pour appuyer cette déconstruction du social. Que vient faire la métaphysique de Tarde dans cette histoire? À supposer que le social n'existe pas, qu'aucune entité n'existe, quel besoin de s'appuyer sur une métaphysique pour le dire? Et qui plus est sur la métaphysique d'un sociologue? La réponse n'est pas énigmatique, elle est même simple. Si le social n'existe pas, c'est qu'il est, comme tout le reste, en invention perpétuelle. Il fallait une théorie de l'invention remplaçant les conventions et les institutions, partout, en tout lieu. Il fallait que cette invention concerne des «mondes hétérogènes» et reconfigure la cosmologie.

On a souvent retenu que la grande idée de Monadologie et sociologie est de substituer une philosophie de l'avoir à une philosophie de l'être, à tous les niveaux de la nature, du psychique au social (il n'y a pas de césure nature / société). La philosophie, fondée sur l'être, aurait tout séparé par des cloisons substantielles, essentielles. Le caractère premier des choses ne serait pas l'abstraction de l'être, toujours identique à lui-même, mais l'acte d'avoir, la différence se faisant. Cette conception ne prend sens que

1. B. Latour, Changer de société, refaire de la sociologie, N. Guilhot (trad.), Paris, La découverte, 2007, p. 348. 
dans une néo-monadologie généralisée où tout point du réel agit, influe, capte, cherche à posséder et à rayonner:

La possession est le fait universel [...]. Tout être veut non pas s'approprier aux êtres extérieurs, mais se les approprier. Adhérence atomique ou moléculaire dans le monde physique, nutrition dans le monde vivant, perception dans le monde intellectuel, droit dans le monde social, la possession aux formes innombrables ne cesse de s'étendre d'un être aux autres êtres, par un entrecroisement de domaines variés, de plus en plus subtils ${ }^{2}$.

Mais demandons-nous à quoi nous conduit cette radicale substitution? S'il n'y a rien d'autre dans la nature que la différence qui diffère («Exister, c'est différer $\left.{ }^{3}\right)$, que l'action possessive de monade à monade, que «l'ambition immense d'un bout de monde à l'autre " ${ }^{4}$, atome vibrant qui attire l'univers à soi, «animacule prolifique», «roi conquérant " 5 , c'est que l'univers lui-même n'est plus un univers, mais une tapisserie de différences, une multitude, un essaim, une foule de lignes de forces. Une cosmologie inventive se substitue à l'univers statique. Chaque fois qu'une différence est produite, inventée, elle se propage, elle remplit l'univers, elle fait monde. On sait que le modèle de diffusion est chez Tarde de type épidémique et contagieux, innervation de proche en proche. Il tient en trois formes de la répétition universelle, «l'ondulation, la génération, l'imitation», qui sont autant d'instruments de conquête, "d'invasion physique, vitale, sociale ${ }^{6}$.

Ce schéma est encore présenté comme toile de fond de La psychologie économique: invention des différences, propagation par répétition, adaptation et variations.

Qu'il s'agisse donc du monde physique, chimique, vital, astronomique, nous saisissons sous les

formes les plus diverses, cette même expansivité essentielle, cette même ambition propagatrice, [qui n'ont,] pour ainsi dire, rien de commun que ce caractère fondamental. Et, si nous nous demandons ce qu'il y a de commun aussi dans les formes sous lesquelles se manifeste ce même vœu d'expansion universelle, nous devons être étonnés, - à moins que l'habitude ne nous aveugle - de constater qu'elles sont toutes des répétitions. Répétition, la série des ondes lumineuses, électriques, sonores, la gravitation des astres, le tourbillonnement intérieur des molécules. Répétition, le tourbillon vital, la nutrition, la respiration, la

2. G. Tarde, Monadologie et sociologie [1893], Paris, Institut d'édition Sanofi-Synthélabo (Les empêcheurs de penser en rond - (Euvres de Gabriel Tarde, 1), 1999, p. 89-90.

3. Ibid., p. 72.

4. Ibid., p. 95.

5. Ibid.

6. Ibid., p. 96 . 
circulation, toutes les fonctions organiques, à commencer par la génération qui les comprend toutes. Répétition, le langage, la religion, le savoir, l'éducation, le travail, toutes les activités sociales, en un seul mot l'imitation?.

Et le même texte de conclure:

Un autre rapprochement que nous apercevons facilement entre les mondes comparés par nous, c'est que les choses qui s'y répètent, mouvements périodiques, types vivants, inventions ou découvertes, sont des thèmes, c'est-à-dire des harmonies fécondes en harmonies nouvelles, des adaptations susceptibles de variations. Des adaptations qui se répètent pour se varier, des nouveautés qui se reproduisent pour se renouveler, tel est le spectacle que nous donne l'Univers envisagé sous tous ses aspects ${ }^{8}$.

Ainsi, l'univers contient des mondes hétérogènes, tous brodés sur des différences et des répétitions. La seule question est de savoir comment la différence s'invente, comment elle crée des plans de différenciation d'une strate à l'autre, comment elle engendre des possibilités nouvelles, produit des variations, ou se stabilise par homogénéisation. En tout cas, c'est ce processus d'invention des différences (l'atome, la cellule, le psychique), avec l'adaptation / variation qui en résulte, qui est à l'œuvre dans toute la nature. Et puisque, selon Tarde, les deux forces cosmologiques sont le désir de conquête et la croyance agrégative («Peut-on nier que le désir et la croyance soient des forces? ${ }^{9}$ ), on a pu parler à ce sujet d'un psychomorphisme. Nous pourrions tout autant parler d'une vision cosmomorphique, car ces deux forces configurent tout le cosmos, saisissent la genèse même de toutes les choses dans ce cosmos.

Il n'est pas innocent que Tarde rappelle ces principes fondamentaux dans un livre consacré à la Psychologie économique. L'économie non plus ne peut s'abstraire du mouvement de différenciation des choses et des êtres, des rapports de désir et de croyance, du rapport subjectif-monde, d'un psychomorphisme qui est un cosmomorphisme. Dans sa Psychologie économique, on voit que les principes de sa cosmologie inventive n'épargnent pas l'économie. On passe de la lumière, de la chaleur, de l'électricité, à la monnaie, en se fondant sur les mêmes principes d'invention et de répétition.

Tout commence par une remise en cause du fondement mathématique de la monnaie, et plus largement des mathématiques comme explication générale. On sait que la monnaie-quantité a servi de base à une économie abstraite. Tarde veut plutôt montrer qu'elle repose nécessairement sur

7. G. Tarde, Psychologie économique, Paris, F. Alcan, 1902, t. I, p. 16. Nous soulignons.

8. Ibid.

9. G. Tarde, Monadologie et sociologie, p. 50. 
des coutumes et des habitudes, qu'il a fallu un nouveau développement des principes subjectifs de désir et de croyance pour qu'elle constitue un nouveau monde qui va capter l'ensemble des désirs. La monnaie-désirabilité universelle, tel serait ce nouveau monde. «Pourquoi ce caractère, qui lui est exclusivement propre, de désirabilité constante, universelle et indéfinie $»^{10}$ ? Comment comprendre que la multiplicité infinitésimale des désirs se mue à un moment précis, particulier, en monnaie, en échangeabilité universelle? Est-ce par calcul de quantification? Certes pas. Ce n'est pas parce que la monnaie était une marchandise universelle, ou une quantité mathématique, que cela s'est passé. Il apparaît plutôt qu'à un moment donné, elle en est venue à condenser dans "un acte de foi universel» des actes de foi multiples (tels les coquillages ou l'ivoire jouant le rôle de monnaie sans usage direct dans les sociétés primitives). Au fond, toute marchandise a pu servir de monnaie, de base du crédit:

La vérité est que toutes les marchandises, avant l'invention de la monnaie proprement dite, ont eu quelque chose de monétaire, chacune d'elles étant signe, jusqu'à un certain point, de toutes les autres ${ }^{11}$.

Autant dire que la monnaie condense des habitudes, des coutumes, des désirs, des croyances, des espérances, et même une "surexcitation des espérances». Cela explique que sa valeur nominale n'ait jamais suivi la production ou la perte de richesses. Dans la physique sociale, on a pu faire de la monnaie une sorte de force première, on a pu avoir l'illusion que, semblablement à la physique, nous avions affaire à une force virtuelle infinie qui circule dans tous les échanges, conversion continuelle d'énergie potentielle en énergie actuelle, mais n'est-ce pas en recouvrant tous les désirs et les croyances qui s'y articulaient que ce mirage s'est imposé ?

Dans de beaux passages, Tarde montre comment la monnaie est un arrachement à la Terre et à la Patrie, une déterritorialisation subite, un cosmopolitisme nouveau, par brisure de «la clôture du désir». Le droit du sol et de la Terre, ainsi que les démocraties terriennes, sont aspirés par cette illimitation du désir, par cette convertibilité du désir, par cet échange des croyances. Le droit ancien reste essentiellement un droit terrien, il passe par «le rapport de l'homme à la Terre». C'est toujours un rapport de possession, d'occupation, d'usage. La monnaie nous place dans une autre dimension. La Terre ne joue plus le rôle d'ancrage, de référence. Par l'inertie de nos représentations, a persisté une conception du capital monétaire en rapport

10. G. Tarde, Psychologie économique, t. I, p. 195. C'est Tarde qui souligne.

11. Ibid., t. II, p. 122. 
avec une Terre immobile qu'on ferait fructifier en l'étendant. Alors que le capital monétaire traduit bien un mouvement cyclique qui sort des gonds d'un ancrage terrestre:

Ne semble-t-il pas, en effet, que c'est en se représentant le capital monétaire comme une sorte de terre indéfiniment stable et permanente, quoique indéfiniment extensible, et, de fait, sans cesse accrue par des capitalisations nouvelles, par des Amériques et des Océanies sans cesse découvertes, qu'on se croit le droit de la faire fructifier tous les ans comme une bonne métairie et de moissonner sa récolte sous forme d'intérêt ${ }^{12}$ ?

Ce qui caractérise la monnaie, c'est à première vue qu'elle n'existe que par rotation, tandis que le capital n'existe lui-même que dans des cycles. Une telle circulation met en défaut tout ancrage dans une Terre immobile. Monnaie et Capital ne sont pas fondiaires ou terrestres. La monnaie résulte du brassage de désirs et de croyances dans une rotation ininterrompue, tandis que le capital monétaire suppose le brassage infiniment varié d'impulsions créatrices, transmis dans des cycles de reproduction, sans aucun immobilisme terrestre. Mais il faut bien voir que, pour Tarde, rotation et cycle qui nous dérobent la Terre immobile sont mal nommés. Il n’y a pas de cercle, de circulation, de rotation, de cycle, tout s'enracine dans l'invention et le rayonnement imitatif. Le monde économique se crée par ce mouvement micro-individuel et étendu des désirs et des croyances. La «série d'imitations d'individu à individu» n'est «nullement circulaire " ${ }^{13}$.

Autant dire encore que la création de la monnaie ne se ramène pas à une loi mathématique, abstraite, elle suppose au contraire un nouveau déploiement des logiques du désir et de la croyance. Son vrai fondement n'est pas la quantité abstraite mais le subjectif:

C’est donc bien à tort que les économistes préoccupés de bâtir leur science sur des fondements tout objectifs ont une prédilection pour le sujet de la monnaie et des finances, où leur rêve, de premier abord, semble se réaliser. En fait, il n'est rien de tel que les phénomènes financiers pour mettre en relief ce qu'il y a d'essentiellement subjectif dans les choses économiques. Par exemple, si les causes de la variation des valeurs de Bourse étaient objectives, comment expliquer cet effondrement ou cette dépression de tous les cours qui a lieu quand une catastrophe ou un événement fâcheux quelconque vient à atteindre un seul de ces titres, mais un de ceux qui donnent le ton aux autres, une rente d'État notamment ${ }^{14}$ ?

12. Ibid., t. I, p. 247 .

13. Ibid., p. 254.

14. Ibid., p. 225. Nous soulignons. 
Dans leur livre sur Tarde, L'économie, science des intérêts passionnés, Bruno Latour et Vincent Antonin Lépinay soulignent avec justesse que c'est une «double abstraction» qui a conduit à la naissance de la théorie économique: celle d'un homme rationnel "sans plus rien d'humain", réduit à des purs calculs d'intérêt, pur automate calculateur, et ensuite d'un homme séparé de toutes ses relations sociales, voisinage, communauté civile, communion religieuse, congrégation, associations... homo œecomonicus desséché, appauvri, vidé de sa chair, censé représenter la quintessence de l'humain. La science quantitative des finances, purement matérielle et objective, détachée de toute considération psychologique et morale, ne prend en compte que cette double abstraction. Comme si, au sein de l'immensité sociale, un continent arctique apparaissait, «un bloc erratique déposé dans le désert de la sociologie », « un continent glacé, l'économie-chose (economy), régie par des lois inflexibles et qui aurait la capacité inouïe de frigorifier toutes les superstructures construites au-dessus d'elle $»^{15}$.

On se méprendrait toutefois si l'on imaginait que Tarde prend ici pour adversaire la quantification de l'humain. Il ne critique pas l'objectivation de l'humain. L'économie prétend quantifier. Soit! Mais elle quantifie peu et mal. Elle est incapable de quantifier le subjectif qui est pourtant le fond même du social: les désirs, les croyances, les séductions, les prestiges, les gloires, les convictions, la vérité, la renommée, le crédit, la crédibilité, le goût, la communication, la foi... Où sont les instruments de valeurs qui mesurent ces réalités et font pourtant partie du social (et qui vont bientôt apparaître comme des disciplines de mesure d'opinion)? Où sont les «tenseurs» et vecteurs du réel? L'économie aurait dû s'étendre «à toutes les évaluations» (expression de Latour et Lépinay), sans se réduire à la valeur argent. En le faisant, elle aurait perdu son caractère abstrait et schématique, rationnel, désertique, parce qu'elle se serait replacée au sein du social. Quantifier l'intersubjectif, l'économie en a été incapable. Elle a souffert d'un grave défaut quantitatif.

C'est la restriction du quantitatif qui a perverti l'économie. Penser l'économie comme une infrastructure mue par des lois calculables, la situer au-dessous du droit, des mœurs, de l'art, aura été une erreur néfaste, que Tarde combattra en la replaçant dans le brassage des actions monadiques interactives, innovantes, désirantes, et donc dans la psychologie. Les concepts fondamentaux de l'économie tels que monnaie, valeur, échange, capital, travail, ne tiennent pas compte des interactions monadiques du réel à leur niveau véritable, c'est-à-dire de l'invention, de la propagation,

15. B. Latour, V. A. Lépinay, L'économie, science des intérêts passionnés, Paris, La découverte, 2008, p. 40. 
de la reproduction. C'est surtout la logique de l'invention et celle de son rayonnement imitatif qui intéressent Tarde. Or, on a trop souvent confondu invention et adaptation. On retrouve la même objection chez Nietzsche, Bergson et Tarde contre le concept d'adaptation. Quand Tarde lit Marx, c'est sur cela qu'il achoppe, le manque de distinction entre invention et reproduction, la trop grande importance donnée aux phénomènes de concurrence relativement à l'invention. Marx découvre le caractère circulaire du capital, du capital-argent, du capital-marchandise, du capital-production. Mais il ne comprend pas l'essence du capital comme capital-germe, capital-invention. Pourtant, c'est cette partie «spirituelle», «subjective», du capital, qui recouvre la fertilité douloureuse des inventions, «le legs des idées indestructibles du génie humain ${ }^{16}$. Elle ne correspond pas au travail non payé de l'esclave, au surtravail non payé de l'ouvrier, mais au «don" qui ne se donne pas, dépense géniale, ingéniosité secrète. Précisément, ce qui se capitalise, ce qui s'accumule, c'est la dépense d'invention. Ce qui est monopolisé, c'est ce capital-là qui ne circule pas, ne s'échange pas, tout en conditionnant absolument les échanges. Tandis que le capital matériel est détruit ou régénéré en permanence (guerres, conflits, échanges). Le capital matériel, Tarde le nomme capital-cotylédon. Il n'est pas nécessaire à l'invention, il consiste dans l'échange des techniques, des marchandises, de la monnaie. Il peut être échangé, donné, prêté, contrairement au capitalgerme. La transmission du capital-germe s'apparente à une émanation qui conserve une partie de sa force initiale, sans aliéner celui qui transmet:

Quant au capital-germe qui consiste en connaissances d'inventions et de découvertes, il ne saurait être, en toute rigueur, ni prêté ni échangé, puisque celui qui le possède ne s'en dessaisit pas en le communiquant à autrui. Il y a là émanation, non aliénation. Il ne saurait être donné non plus, ni volé, pour la même raison. Cependant sa possession exclusive est un bien d'une grande importance souvent, et la perte de ce monopole par le partage de cette subite possession devenue indivise avec autrui, par une communication à titre onéreux ou à titre gratuit, équivaut à un don, à un prêt ou à une vente. En fait, dans les familles primitives, tout ce capital-germe, à savoir les secrets de fabrication, de remèdes, de poisons, etc., est jalousement gardé, héréditairement transmis, ce qui montre le sentiment profond qu'on a de son importance majeure ${ }^{17}$.

En lisant bien Marx, on verrait que lui-même ne répugne pas à attribuer une forme de subjectivité au mouvement du capital:

16. G. Tarde, Psychologie économique, t. I, p. 237.

17. Ibid., p. 258-259. 
Il y a chez Karl Marx parfois une tournure ontologique, j'allais dire mythologique, d'esprit, qui lui vient de Hegel. Le capital, la valeur, sont pour lui des êtres qu'il anime de sa passion et de sa vie ${ }^{18}$.

On verrait aussi que l'on peut interpréter les crises périodiques du capitalisme comme étant en rapport de croyance, de crédit, avec les phases et les déphasages de l'inventivité.

Les périodes de précipitation, de surproduction fiévreuse qui précèdent les crises et les préparent, sont dues, en majeure partie, aux excès de confiance suscités par les nouvelles inventions qui révolutionnent les industries. Il ne faudrait donc voir, d'après cela, dans la série périodique des crises depuis cent ans, qu'un signe manifeste de notre inventivité moderne; et, si celle-ci est «activée par l'expansion du régime capitaliste», il n'y aurait pas lieu de tant maudire le capitalisme. À ce point de vue, la périodicité presque régulière des crises pendant le $\mathrm{XIX}^{\mathrm{e}}$ siècle s'expliquerait par la fécondité même du génie industriel de notre temps, qui répondrait toujours au moment voulu, par de nouvelles poussées d'inventions, à l'appel d'une nouvelle vague d'optimisme général prête à se soulever après une dépression de pessimisme... D’où on pourrait conclure que, inévitablement, cette périodicité doit aller s'effaçant quand l'invention se raréfiera et s'épuisera, ce qui arrivera un jour ou l'autre ${ }^{19}$.

De même, lorsque Tarde lit Darwin, il lui objecte d'avoir trop mis l'accent sur les conditions de la concurrence vitale. De là vient qu'on ait confondu trop souvent le véritable mouvement de création du vivant, «l'hybridité féconde», avec la concurrence et l'opposition interindividuelle :

Le grand mérite de Darwin aura été de montrer la portée méconnue de la tendance des vivants à leur multiplication indéfinie, et de suivre les conséquences qui en découlent, telles que la concurrence vitale et le croisement des espèces. Son erreur, s'il m'est permis d'apprécier ce grand homme en m'autorisant d'autres grands naturalistes, me semble avoir été d'appuyer beaucoup plus sur la concurrence vitale, forme biologique de l'opposition, que sur le croisement et l'hybridité, formes biologiques de l'adaptation et de l'harmonie. Une fonction aussi importante que la production d'une nouvelle espèce ne saurait être une fonction continue et quotidienne, alors que la simple production d'un individu nouveau, la génération, est une fonction intermittente. Un phénomène exceptionnel, et non pas un phénomène journalier, doit être à la base de cette nouveauté spécifique. Et, je suis de l'avis de Cournot, une hybridité féconde, par exception, est bien plus propre qu'une accumulation héréditaire de petites variations avantageuses, par

18. G. Tarde, Psychologie économique, t. II, p. 140.

19. Ibid., p. 141. 
concurrence et sélection, à expliquer la formation de nouveaux types vitaux. Encore faut-il reconnaître que l'on indique ainsi les conditions seulement du merveilleux phénomène, et non ses causes, qui restent le secret des ovules fécondés où il s'opère.

Darwin n'a point paru s'apercevoir que les variations individuelles qu'il postule, comme les données et les matériaux avec lesquels la sélection bâtirait de nouvelles espèces, sont elles-mêmes autant de petites créations, de petites réadaptations du type ancien, dont la genèse n'est guère moins merveilleuse que celle d'un type nouveau, auquel chacune d'elles vise, et est du même ordre, au fond. En sorte que la genèse des innovations vitales est pour ainsi dire, postulée par cela même qui est censé l'expliquer ${ }^{20}$.

On devrait dire au contraire que les variations individuelles sont surtout des créations, des inventions, des innovations vitales. Cette logique de la création n'est pas saisie chez Marx et Darwin. Il faudrait descendre au niveau des inventions et des imitations, dans le Mystère secret de la création, suivre le labyrinthe de la cosmologie inventive au lieu d'en rester à des oppositions vitales ou des oppositions de classes.

Partout est manquée la dimension subjective de l'invention et du désir. Ou de la croyance et de la foi. Lorsqu'en économie, on vient se placer d'abord sur le plan des échanges, et non sur celui de la production inventive, il est visible que cette dimension subjective est à nouveau oblitérée. On retrouve la même erreur chez tous les économistes. Ils font comme si le fondement de l'économie était l'échange. Mais, pour Tarde, le fait économique fondamental est le crédit, source «de la vie productive», et non pas l'échange:

J'ai encore à faire remarquer à propos du capital que sa véritable source, dès les plus anciens débuts de l'évolution économique, est un acte de foi et de confiance, premier embryon du crédit, qui est, manifestement, l'âme de la vie productrice des sociétés civilisées. On n'a dit que la moitié de la vérité quand on a vu dans le contrat d'échange le fait économique essentiel et initial. L'échange, à vrai dire, ne favorise et ne développe directement que la consommation. L'agent direct de la production est un autre contrat, non moins initial, non moins fondamental, le contrat de prêt. Par l'échange, on se rend service l'un à l'autre, mais en se défiant l'un de l'autre: donnant donnant; par le prêt, on se confie ${ }^{21}$.

Le fond même de l'économie est le crédit. Les grandes crises économiques sont toujours des crises du crédit, de la confiance, de la croyance autour de ce qu'on peut confier ou non. Là encore, le principal est subjectif. Il est visible

20. Ibid., t. I, p. 17 .

21. Ibid., p. 256. 
que la croyance, la foi économique, le credo dogmatique des acteurs sont une action subjective qui ne saurait être séparée du jeu économique lui-même.

La non-compréhension de la dimension psychologique de l'économie explique que l'on ait tant de mal à comprendre le mécanisme des crises économiques qui sont réduites à des crises de surproduction ou à des crises financières. Ainsi la dernière crise financière que Frédéric Lordon analyse de la sorte: «acharnement dogmatique envers et contre les infirmations du réel ", «constance dans l'aberration » du choix des solutions de crise. La crise est bien la «combinaison hétéroclite de stratèges opportunistes et de croyants au premier degré», elle est une forme de folie des certitudes néolibérales, une comédie des arrangements au plus haut sommet de l'Europe. Le problème c'est bien au fond ce que les acteurs croient. L'obstination des acteurs économiques à suivre aveuglément les schémas les plus éculés et inefficaces est stupéfiante. Frédéric Lordon conclut: «si ce n'est l'esprit de Laurel et Hardy qui règne aujourd'hui sur l'Europe, c'est peut-être alors celui de saint Augustin: Credo quia absurdum (j'y crois parce que c'est absurde) $»^{22}$.

La partie subjective de l'économie n'a cessé d'être étouffée. Par quel miracle l'économie a-t-elle pu se muer en science désintéressée de l'intérêt quand tout repose sur des persuasions et des «passions dominantes»? (c'est, au fond, ce que Tarde n'aura cessé de dire). Il y a, selon Tarde, un fond irrationnel de l'économie pris dans des jeux «d'une intensité inouïe» de conquête, de lutte, de possession. En temps de crise, les marchés financiers échangent principalement de la confiance, du crédit, de la croyance, de la passion. «Si l'on fait abstraction de ces convictions et de ses passions dominantes, qui, sourdes, ou conscientes, sont les forces sociales individuelles, qu'explique-t-on en économie politique?», demande Tarde ${ }^{23}$.

Quand Frédéric Lordon pense l'économie à partir de captures de désirs, d'enrôlement, d'échanges de croyances, il semble se rattacher sans le voir à Tarde autant qu'à Spinoza. La différence avec Tarde est qu'il maintient «la présence des structures au sein des sujets mais sous forme de dispositions, de désirs, de croyances et d'affects ${ }^{24}$. Il pense en somme mieux appréhender l'effet de la structure en la dédoublant, capitalisme objectif d'un côté, effets dans la configuration subjective et désirante de l'autre. Il accorde une place importante aux désirs, croyances et affects, mais peut-être encore trop à une structure qui les domine.

22. F. Lordon, «Sur le toboggan de la crise européenne», Le Monde diplomatique, décembre 2011, p. 1 et 12-13.

23. G. Tarde, Psychologie économique, t. II, p. 34.

24. F. Lordon, Capitalisme, désir et servitude. Marx et Spinoza, Paris, La fabrique, 2010, p. 29. 
Le thème de la croyance, plutôt que celui de la vérité, passe au premier plan chez de nombreux auteurs, de Nietzsche aux frères James, de Tarde à Deleuze. L'ouvrage Fictions du pragmatisme de David Lapoujade éclaire très bien le couple charité/volonté, central dans les écrits des frères James, en montrant que ces deux auteurs ne se situent plus à l'âge des crises monétaires mais à celui de la circulation d'argent entre des trusts, les bien nommés, c'est-à-dire à ce nouvel âge de l'équivalence entre capital et confiance, dette et crédit. Alors il ne s'agit plus de tromper la confiance des individus comme chez Melville (voir la préface au roman de Melville, L'escroc à la confiance), mais de les déposséder de leur puissance vitale: la dette illimitée ou le "monde des Zombies", c'est le monde de la pure dépossession de $\operatorname{soi}^{25}$. La seule justification morale du monde étant celle du crédit-dette illimitée, le capitalisme produit un vampirisme qui exténue les vies, les dépossède d'elles-mêmes. Tout est affaire de possession, même l'identité personnelle, même le moi, toutes les vies peuvent être subjuguées, hypnotisées, vampirisées. Comment leur soustraire de la puissance? D'où le surgissement d'une volonté terrible, crispée, négative, qui surplombe les vies. Lawrence disait de l'Amérique dans une lettre: «Tout est fondé sur la volonté. Une puissante volonté négative semble s'opposer à toute vie spontanée... Ils ne font pas confiance à la vie tant qu'ils ne la dominent pas ». La volonté et la charité s'érigent en principe de domination et traduisent le fond même du capitalisme: "Ce sont les deux mors de l'étau, une négation volontaire et une abnégation caritative, l'effroyable tour de vis ${ }^{26}$. Si bien que la seule question du salut dans le capitalisme est: comment se sauver sans être sauvé par charité, comment entrer dans un système de possession sans être dépossédé de soi ? Il y a chez les frères James une psychologie du capitalisme qui rejoint celle de Tarde.

L'avantage de la métaphysique de Tarde est de placer l'invention et la croyance au centre d'une cosmologie d'interférences subjectives. Dans cette vision cosmomorphique tout élément différentiel entre dans une intégrale qui implique les autres, tout élément, qu'il soit naturel, biologique, technique ou politique, résonne dans tous les autres, produit des effets sur eux et en subit en retour. Le fourmillement des influences infinitésimales crée des lignes de devenir capables de courber l'histoire ou l'économie, la science ou la religion. Aucune instance ne commande, ni dans la nature, ni dans la société, aucun Dieu, aucune loi providentielle, aucune intelligence, aucune

25. D. Lapoujade, in H. Melville, "Bartleby le scribe" - "Billy Budd, marin" et autres romans, P. Jaworski (éd.), D. Lapoujade, H. Parker (coll.), Paris, Gallimard (CEuvres, 4 - Bibliothèque de la Pléiade, 559), 2010.

26. D. Lapoujade, Fictions du pragmatisme, Paris, Minuit, 2008, p. 217. 
raison. Le réel laisse place aux inventions les plus hétéroclites, bizarres, voire grimaçantes ( «le caractère bizarre et grimaçant de la réalité » ${ }^{27}$ ), comme aux plus étonnantes, "féériques», bigarrées. L'action incessante de ces poussées infinitésimales sillonnant en tout sens l'univers modifie l'espace et le temps, produit des conjonctures nouvelles, des microcosmes et des milieux étranges, des fédérations inédites. On comprend que Bruno Latour ait pu être fasciné par cette cosmologie inventive. Car c'est bien l'invention et la croyance qui deviennent le foyer de toutes choses. Elles seules forment des mondes, y compris le monde économique.

Pierre Montebello

Université Toulouse 2

27. G. Tarde, Monadologie et sociologie, p. 93. 